\title{
Non-universal critical properties of a symmetrical binary fluid mixture
}

\author{
O.V.Patsahan, R.S.Melnyk, M.P.Kozlovskii \\ Institute for Condensed Matter Physics \\ of the National Academy of Sciences of Ukraine, \\ 1 Svientsitskii Str., 79011 Lviv, Ukraine
}

Received August 21, 2000

\begin{abstract}
We study non-universal critical properties of a symmetrical mixture using the recently proposed approach and the method of layer-by-layer integration of a partition function. Both the gas-liquid critical point properties (temperature and density) and the fluid-fluid demixing temperature of a symmetrical hard-sphere square-well mixture are calculated depending on its microscopic parameters: the parameter $r$ measuring the strength of interactions between the particles of dissimilar and similar species and the parameter $\lambda$ measuring the width of the potential well.
\end{abstract}

Key words: non-universal critical properties, gas-liquid critical point, fluid-fluid demixing, binary mixture

PACS: 05.70.Fh, 05.70.Jk, 65.10.+h

\section{Introduction}

It is known that the topology of the phase diagram of a one-component fluid is relatively insensitive to the microscopic properties of the molecules and exhibits a gas-liquid first-order line terminating at a critical point. The situation is significantly different in the case of binary mixtures where a variety of phase behaviour is possible according to the relative sizes of the molecules and the strengths of their interactions.

A comparison between theory and experiments in the critical region of simple fluids and their mixtures has shown that the available liquid state theory and field theory methods leave several open questions, mostly regarding non-universal quantities. One of them is the accurate determination of the critical parameters.

In this paper we shall study the critical properties of the binary symmetrical mixture (BSM). The BSM is the model mixture in which the two pure components " $a$ " and " $b$ " are identical and only interactions between the particles of dissimilar species differ. Due to the symmetry of such a system, its critical concentration is $x_{\mathrm{c}}=0.5$. Notwithstanding its simplicity, the BSM exhibits all three types of two-phase equilibrium which are observed in real binary fluids: gas-liquid, liquid- 
liquid and gas-gas equilibria. This model have recently been studied using Monte Carlo (MC) simulations in [1-4], where both the gas-liquid (GL) and fluid-fluid (FF) critical point properties were calculated for a symmetrical mixture of hard spheres interacting via square-well potentials.

We use the approach proposed recently for a non-symmetrical binary fluid mixture (the system of different size particles interacting via different potentials) [5]. This approach is based on the functional representation of a partition function in the collective variables (CV) space [6]. The proposed approach allows us to solve the following tasks [7-9,5]: (1) to take into account both the short-range and the long-range interactions simultaneously; (2) to introduce the order parameter in a natural way; (3) to obtain the effective Ginsburg-Landau-Wilson (GLW) Hamiltonian explicitly related to the microscopic properties; (4) to integrate the partition function in the neighbourhood of the phase transition point taking into account the renormalization group symmetry.

\section{Functional of the grand partition function of a binary mix- ture. The HLW Hamiltonian}

We consider a binary fluid mixture consisting of $N_{a}$ particles of species "a" and $N_{b}$ particles of species "b". The interaction potential between particles can be expressed as a sum of two terms:

$$
U_{\gamma \delta}(r)=\Psi_{\gamma \delta}(r)+\Phi_{\gamma \delta}(r)
$$

where $\Psi_{\gamma \delta}(r)$ is a potential of a short-range repulsion and $\Phi_{\gamma \delta}(r)$ is an attractive part of the potential. The thermodynamic and structural properties of a system with pair interaction $\Psi_{\gamma \delta}(r)$ (reference system $(\mathrm{RS})$ ) are assumed to be known from some other theory.

A functional of the grand partition function (GPF) of the binary homogeneous system in the $\mathrm{CV}$ method with a $\mathrm{RS}$ can be presented as a product of two factors $[5]$ :

$$
\Xi=\Xi_{0} \Xi_{1}
$$

where $\Xi_{0}$ is the GPF of the RS. $\Xi_{1}$ is the part of the GPF which is written in the CV space:

$$
\begin{aligned}
\Xi_{1}=\int & (\mathrm{d} \rho)(\mathrm{d} c) \exp \left\{\beta \mu_{1}^{+} \rho_{0}+\beta \mu_{1}^{-} c_{0}\right. \\
& \left.-\frac{\beta}{2 V} \sum_{\mathbf{k}}\left[\tilde{V}(k) \rho_{\mathbf{k}} \rho_{-\mathbf{k}}+2 \tilde{U}(k) \rho_{\mathbf{k}} c_{\mathbf{k}}+\tilde{W}(k) c_{\mathbf{k}} c_{-\mathbf{k}}\right]\right\} J(\rho, c) .
\end{aligned}
$$

Chemical potentials $\mu_{1}^{+}$and $\mu_{1}^{-}$are determined from the conditions:

$$
\frac{\mathrm{d} \ln \Xi_{1}}{\mathrm{~d} \beta \mu_{1}^{+}}=\left\langle N_{a}\right\rangle+\left\langle N_{b}\right\rangle=\langle N\rangle, \quad \frac{\mathrm{d} \ln \Xi_{1}}{\mathrm{~d} \beta \mu_{1}^{-}}=\left\langle N_{a}\right\rangle-\left\langle N_{b}\right\rangle .
$$


The functions $\tilde{V}(k), \tilde{W}(k)$ and $\tilde{U}(k)$ are combinations of Fourier transforms of the initial interaction potentials $\tilde{\Phi}_{\gamma \delta}(k)$ :

$$
\begin{aligned}
\tilde{V}(k) & =\left(\Phi_{a a}(k)+\Phi_{b b}(k)+2 \Phi_{a b}(k)\right) / 2, \\
\tilde{U}(k) & =\left(\Phi_{a a}(k)-\Phi_{b b}(k)\right) / 2 \\
\tilde{W}(k) & =\left(\Phi_{a a}(k)+\Phi_{b b}(k)-2 \Phi_{a b}(k)\right) / 2 .
\end{aligned}
$$

$J(\rho, c)$ is a Jacobian for the transition to the $\mathrm{CV} \rho_{\mathbf{k}}, c_{\mathbf{k}}$ averaged on the RS:

$$
J(\rho, c)=\int(\mathrm{d} \nu)(\mathrm{d} \omega) \exp \left\{\mathrm{i} 2 \pi \sum_{\mathbf{k}}\left(\omega_{\mathbf{k}} \rho_{\mathbf{k}}+\nu_{\mathbf{k}} c_{\mathbf{k}}\right)+\sum_{n \geqslant 1} \sum_{i_{n} \geqslant 0} D_{n}^{\left(i_{n}\right)}(\omega, \nu)\right\},
$$

where

$$
\begin{aligned}
D_{n}^{\left(i_{n}\right)}(\omega, \nu)= & \frac{(-\mathrm{i} 2 \pi)^{n}}{n !}\left(\frac{1}{2}\right)^{n / 2} \sum_{\mathbf{k}_{1} \ldots \mathbf{k}_{n}} \mathcal{M}_{n}^{\left(i_{n}\right)}\left(\mathbf{k}_{1}, \mathbf{k}_{2}, \ldots, \mathbf{k}_{n}\right) \\
& \times \nu_{\mathbf{k}_{1}} \ldots \nu_{\mathbf{k}_{i_{n}}} \omega_{\mathbf{k}_{i_{n}+1}} \ldots \omega_{\mathbf{k}_{n}} \delta_{\mathbf{k}_{1}+\ldots+\mathbf{k}_{n}} .
\end{aligned}
$$

The variables $\omega_{\mathbf{k}}$ and $\nu_{\mathbf{k}}$ are conjugated to the variables $\rho_{\mathbf{k}}$ and $c_{\mathbf{k}}$, respectively. The cumulants $\mathcal{M}_{n}^{\left(i_{n}\right)}\left(\mathbf{k}_{1}, \mathbf{k}_{2}, \ldots, \mathbf{k}_{n}\right)$ are linear combinations of the n-particle partial structure factors of the RS (see [10] ).

$\mathrm{CV} \rho_{\mathbf{k}}$ and $c_{\mathbf{k}}$ are connected with the total density fluctuation modes and the relative density (or concentration) fluctuation ones, respectively.

In order to derive the effective GLW Hamiltonian we perform the following program:

- we determine the CV connected with the order parameter by diagonalizing the square form in (2.1);

- after integration over irrelevant CV (which do not include the variable connected with the order parameter) with the Gaussian density measure we construct the GLW Hamiltonian.

As a result we obtain (within the framework of the $\phi^{4}$-model):

$$
\Xi=C \int \exp \left[E_{4}(\eta)\right](\mathrm{d} \eta)^{N_{B}}
$$

where the GLW Hamiltonian has the form:

$$
\begin{aligned}
E_{4}(\eta)= & h \eta_{0}-\frac{1}{2\left\langle N_{B}\right\rangle} \sum_{\vec{k}} d_{2}(k) \eta_{\vec{k}} \eta_{-\vec{k}} \\
& -\frac{a_{4}}{4 !\left\langle N_{B}\right\rangle^{3}} \sum_{\vec{k}_{1} \ldots \vec{k}_{4}} \eta_{\vec{k}_{1}} \eta_{\vec{k}_{2}} \eta_{\vec{k}_{3}} \eta_{\vec{k}_{4}} \delta_{\vec{k}_{1}+\vec{k}_{2}+\vec{k}_{3}+\vec{k}_{4}}, \quad\left|\vec{k}_{i}\right|<B, \\
& d_{2}(k)=a_{2}+P(k),
\end{aligned}
$$


$P(k)$ is a linear combination of the functions $\tilde{V}(k), \tilde{W}(k)$ and $\tilde{U}(k)$. The behaviour of function $P(k)$ in the neighbourhood of the phase transition point is similar to the behaviour of the initial potentials $\tilde{\Phi}_{\gamma \delta}(k)$. The radius $B$ in $(2.4)$ is found from the condition $P(k=B)=0$ and it is considered as the size of the first Brillouin zone of a certain block lattice.

$\eta_{\vec{k}=0}$ is the CV connected with the order parameter in the case of the GL critical point, as well as in the case of the mixing-demixing phase transition [11]:

$$
\eta_{0}=B(0) \rho_{0}+D(0) c_{0}
$$

where the coefficients $B(0)$ and $D(0)$ are certain functions of the microscopic parameters, temperature, density and concentration of the system (they can also be expressed by the thermodynamic relations). As it is seen from (2.6) both the GL and mixing-demixing phase transitions are accompanied by the total density fluctuations as well as by the relative density (or concentration) fluctuations. The contribution from each type of these fluctuation processes changes along the critical curve. In our approach we can evaluate such contributions at each point of the critical curve.

As it is seen from $(2.4), E_{4}(\eta)$ has the form analogous to the basic density measure of the $3 D$ Ising model in an external field [7]. But the main difference is the dependence of coefficients $h, a_{2}$ and $a_{4}$ on the microscopic parameters, temperature, density and concentration of the system.

\section{GL and FF critical properties of the symmetrical square-well mixture}

Using the expression for the GLW Hamiltonian (2.4)-(2.5) and the method of layer-by-layer integration proposed for the 3D Ising model [7] we calculate the critical parameters of the model binary symmetrical mixture.

We consider a symmetrical hard-sphere square-well binary mixture. The interaction in the system is described by the potential

$$
U_{\gamma \delta}(r)=\left\{\begin{array}{cc}
\infty, & r<\sigma \\
-\epsilon_{\gamma \delta}, & \sigma \leqslant r<\lambda \sigma \\
0, & r \geqslant \lambda \sigma
\end{array}\right.
$$

where $\sigma$ is a hard-sphere diameter, $\lambda$ is a range of the potential, and $\epsilon_{\gamma \delta}$ is a welldepth of the interaction between the particles of types $\gamma$ and $\delta$. The square-well potential is the simplest model which includes the presence of attractive and repulsive forces. For a symmetrical mixture $\epsilon_{a a}=\epsilon_{b b}=\epsilon \neq \epsilon_{a b}$. We introduce the free model parameter $r$ measuring the strength of the interaction between the particles of dissimilar and similar species: $r=\epsilon_{a b} / \epsilon$. The case $r=1$ corresponds to a onecomponent fluid. In our formalism a completely analytical treatment for general $\lambda$ is possible. 


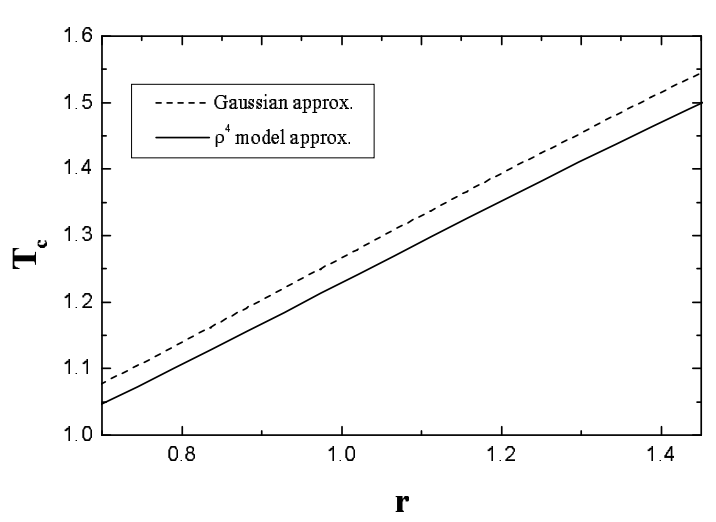

Figure 1. The GL critical temperature $\left(T_{\mathrm{c}}=k T / \epsilon\right)$ as a function of the microscopic parameter $r$ at $\lambda=1.5$.

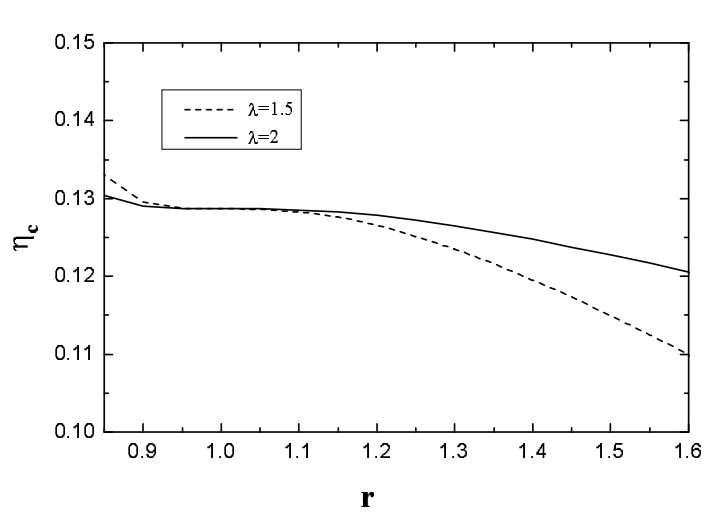

Figure 2. The critical density $\left(\eta_{\mathrm{c}}=\right.$ $\left.\pi \rho_{\mathrm{c}} \sigma^{3} / 6\right)$ as a function of the microscopic parameter $r$

We split the potential $U_{\gamma \delta}(r)$ into short- and long-range parts using the WeeksChandler-Andersen partition [12]. As a result, we have:

$$
\begin{aligned}
& \Psi_{\gamma \delta}(r)=\left\{\begin{array}{cc}
\infty, & r \leqslant \sigma \\
0, & r>\sigma
\end{array},\right. \\
& \Phi_{\gamma \delta}(r)=\left\{\begin{array}{cc}
-\epsilon_{\gamma \delta}, & 0 \leqslant r \leqslant \lambda \sigma \\
0, & r>\lambda \sigma
\end{array} .\right.
\end{aligned}
$$

In this case the RS is a one-component hard-sphere system with the diameter $\sigma$ (see $(3.1)$ ). The cumulants $\mathcal{M}_{n}^{\left(i_{n}\right)}(0, \ldots, 0)$ are calculated according to the formulas given in [13]. The Percus-Yevick approximation is used for $\mathcal{M}_{2}(0)$.

We shall not dwell on the layer-by-layer integration method in this paper. We shall only outline its main idea.

The essence of the method consists in a subsequent integration over the layers of the CV space, beginning with the $\mathrm{CV} \rho_{\vec{k}}$ which correspond to short-wavelength fluctuations. Integration is performed according to the following scheme. The region $(0, B)$ is divided into the intervals $\left(B_{1}, B\right), \ldots,\left(B_{i+1}, B_{i}\right), \ldots$, by a division parameter $S\left(B_{n}=B / S^{n}\right)$. Each interval corresponds to a layer of subscripts $\mathbf{k}$ in the Brillouin zone and each layer of subscripts $\mathbf{k}$ corresponds to a layer in the phase space of $\mathrm{CV} \rho_{\mathbf{k}}$. Integrating gradually over the layers we get a block lattice sequence with an appropriately growing block period and with the Hamiltonian corresponding to each block. Each Hamiltonian is characterized by a set of the coefficients $d_{2}, a_{4} ; d_{2}^{(1)}, a_{4}^{(1)} ; d_{2}^{(2)}, a_{4}^{(2)}$, etc. For the sequence of the block Hamiltonians $\left\{d_{2}^{(n)}, a_{4}^{(n)}\right\}$ the renormalization group symmetry holds and the fixed point is of a saddle type. As a result of the integration we obtain recursion relations for the coefficients of the GLW Hamiltonian. The analysis of these relations yields the equation for the critical temperature:

$$
A(\beta \tilde{V}(0))^{2}+B(\beta \tilde{V}(0))+D=0
$$


Table 1. Critical parameters of the symmetrical hard-sphere square-well mixture (FF demixing temperatures are calculated at $\eta=0.268$ ).

\begin{tabular}{ccccc}
\hline$r$ & $\lambda$ & $T_{\mathrm{c}}^{\mathrm{CV}}(\mathrm{Gauss})$ & $T_{\mathrm{c}}^{\mathrm{CV}}\left(\phi^{4}\right)$ & $T_{\mathrm{c}}^{\mathrm{MC}}$ \\
\hline 0 & 1.5 & $3.618(\mathrm{FF})$ & $2.908(\mathrm{FF})$ & $2.770(44)^{a}(\mathrm{FF})$ \\
0.72 & 1.5 & $1.09(\mathrm{GL})$ & $1.058(\mathrm{GL})$ & $1.06(1)^{b}(\mathrm{GL})$ \\
1.0 & 2.0 & $3.002(\mathrm{GL})$ & $2.934(\mathrm{GL})$ & $2.684(51)^{c}(\mathrm{GL})$ \\
\hline \multicolumn{5}{c}{ (a) Reference [2], (b) Reference [3], (c) Reference [4]. }
\end{tabular}

where

$$
\begin{aligned}
A & =1-f_{0}-R^{(0)} \sqrt{\varphi_{0}} \\
B & =-a_{2} \\
D & =a_{4} R^{(0)} / \sqrt{\varphi_{0}} .
\end{aligned}
$$

$f_{0}, \varphi_{0}$ are coordinates of the reduced fixed point, $R^{(0)}$ is a universal function of parameter $S$. We choose $S=3.4252$ and the values of $f_{0}, \varphi_{0}, R^{(0)}$ corresponding to it are taken from [14].

\subsection{GL critical point of the symmetrical square-well mixture}

We consider the hard-sphere squarewell binary fluid in the neighbourhood of the GL critical point. In this case the GLW Hamiltonian has the same form as (2.4)-(2.5) but the main quantities are reduced to the following ones [13]:

$$
\eta_{\vec{k}}=\rho_{\vec{k}}, \quad P(k)=\tilde{V}(k), \quad h=\mu^{*} .
$$

From the condition

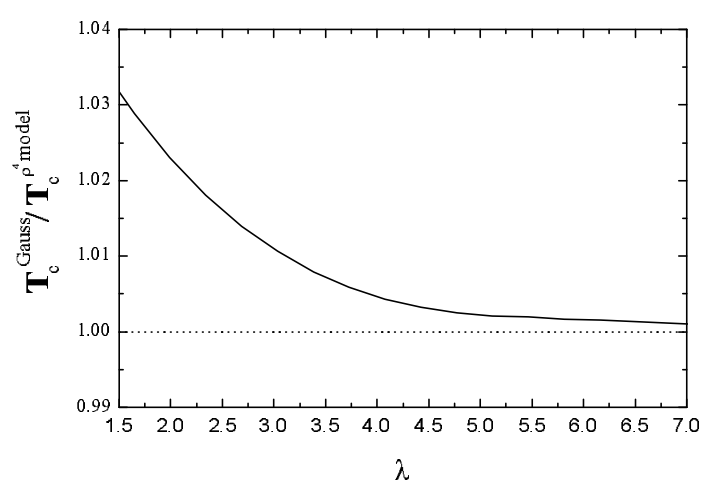

$$
\mu^{*}=0
$$

we obtain the equation for the critical

Figure 3. The GL critical temperature as a function of $\lambda(r=1.2)$.

density of the system. The solutions of equations (3.3)-(3.4) are found numerically using a self-consistent procedure. The results are shown in figures 1 (critical temperature) and 2 (critical density). Figure 3 depicts the dependence of the GL critical temperature on the width of the potential well $\lambda$ for $r=1.2$.

\subsection{FF demixing of the symmetrical square-well mixture.}

We study the FF demixing critical temperature as a function of parameter $r$ and dimensionless density $\eta$. For the symmetrical mixture in the neighbourhood of the 


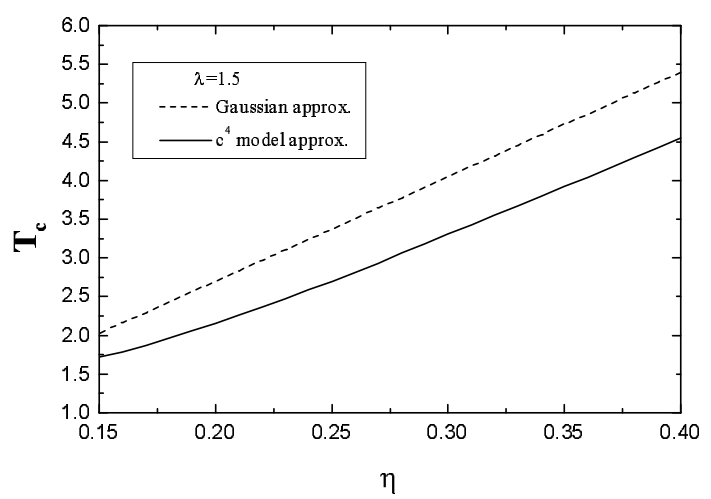

Figure 4. The FF critical curve of the symmetrical square well mixture $(r=0)$.

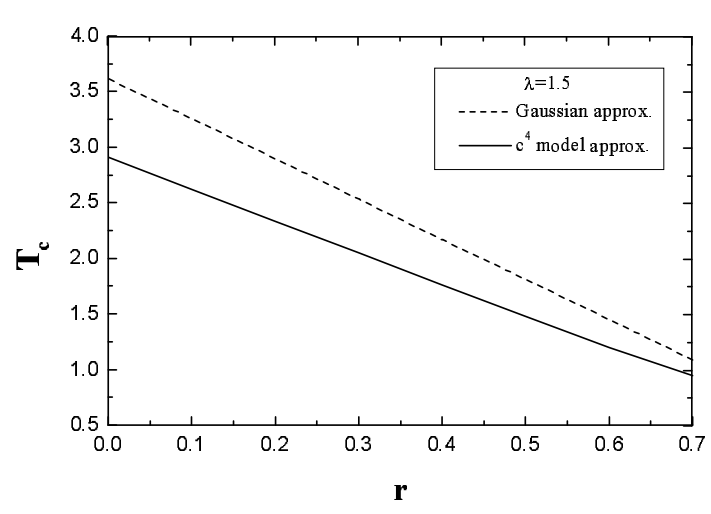

Figure 5. The FF demixing temperature $\left(T_{\mathrm{c}}=k T / \epsilon\right)$ as a function of the microscopic parameter $r$ at $\eta=0.268$.

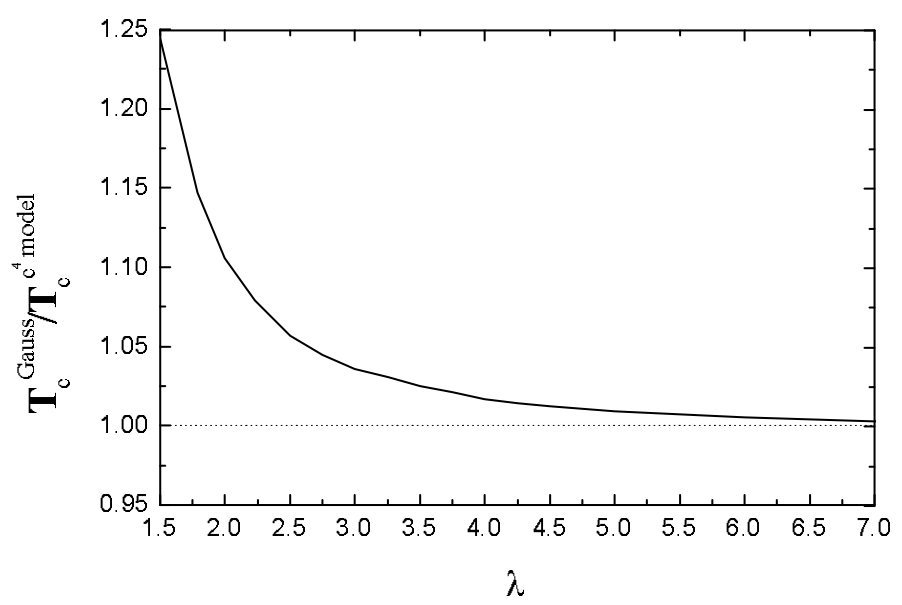

Figure 6. The FF demixing temperature as a function of $\lambda(r=0.0, \eta=0.268)$.

FF demixing point the main quantities from (2.4)-(2.5) are reduced to the following ones:

$$
\eta_{\vec{k}}=c_{\vec{k}}, \quad P(k)=\tilde{W}(k), \quad h=0 .
$$

As a result we obtain a critical line of the $\mathrm{FF}$ demixing points $T_{\mathrm{c}}(\eta)$ where $\eta=\pi \rho \sigma^{3} / 6$ is the reduced density (see figure 4 ). The dependence of the FF demixing temperature on the parameter $r$ is shown in figure 5 .

Figure 6 displays the results for FF demixing temperatures depending on the width of the potential well $\lambda$ for $r=0$. We compare our results with the MC simulation data in table 1.

\section{References}

1. Recht J.R., Panagiotopoulos A.Z. // Mol. Phys., 1993, vol. 80, No. 4, p. 843-852. 
2. de Miguel E., del Rio E.M., Telo da Gama M.M. // J. Chem. Phys., 1995, vol. 103, No. 14 , p. $6188-6196$.

3. Wilding N.B. // Phys. Rev. E, 1998, vol. 58, No. 2, p. 2201-2212.

4. de Miguel E. // Phys. Rev. E, 1997, vol. 55, No. 2, p. 1347-1354.

5. Patsahan O.V. // Physica A, 1999, vol. 272, p. 358-375.

6. Yukhnovskii I.R., Holovko M.F. Statistical Theory of Classic Equilibrium Systems. Kyiv, Naukova dumka, 1980 (in Russian).

7. Yukhnovskii I.R. Phase Transitions of the Second Order. The Collective Variables Method. Singapore, World Scientific, 1987; Yukhnovskii I.R. // Rivista del Nuovo Cim., 1989, vol. 12, No. 1, p. 1-120.

8. Yukhnovskii I.R. // Physica A, 1999, vol. 168, No. 3, p. 999-1020.

9. Patsahan O.V., Yukhnovskii I.R. // Theor. Math. Phys., 1990, vol. 83, p. 387-397.

10. Yukhnovskii I.R., Patsahan O.V. // J. Stat. Phys., 1995, vol. 81, No. 3/4, p. 647-672.

11. Patsahan O.V. // Condens. Matter Phys., 1999, vol. 2, No. 2(18), p. 235-241.

12. Andersen H.C., Chandler D., Weeks J.D. // J. Chem. Phys., 1972, vol. 56, p. 3812.

13. Patsahan O.V., Kozlovskii M.P., Melnyk R.S. // J. Phys.: Condens. Matter, 2000, vol. 12, p. 1595-1612.

14. Kozlovskii M.P., Yukhnovskii I.R. Method for the self-consistent description of the critical behaviour of thre three-dimensional Ising-like system at a microscopic level. Preprint of the Institute for Condensed Matter Physics, ICMP-97-25U, Lviv, 1997, 95 p. (in Ukrainian).

\title{
Неуніверсальні критичні властивості симетричної флюїдної суміші
}

\author{
О.В.Пацаган, Р.С.Мельник, М.П.Козловський \\ Інститут фізики конденсованих систем НАН України, \\ 79011 Львів, вул. Свєнціцького, 1 \\ Отримано 21 серпня 2000 р.
}

Ми вивчаємо неуніверсальні критичні властивості симетричної бінарної суміші, використовуючи недавно запропонований підхід і метод пошарового інтегрування статистичної суми. Для симетричної суміші твердих сфер, які взаємодіють з потенціалом прямокутної ями, обчислені властивості критичної точки газ-рідина (температура і густина) і температура незмішування флюїд-флюїд залежно від мікроскопічних параметрів: параметра $r$, який вимірює силу взаємодії між частинками неподібних і подібних сортів, і параметра $\lambda$, який вимірює ширину потенціальної ями.

Ключові слова: неуніверсальні критичні властивості, критична точка газ-рідина, незмішування флюїд-флюїд, бінарна суміш

PACS: $05.70 . F h, 05.70 . J k, 65.10 .+h$ 